\title{
Estimación del valor de uso directo del suelo en el ámbito del Proyecto Piloto de Forestación (PPF), Granja Porcón, Cajamarca
}

\author{
Estimated value of direct soil use in the field of the Afforestation Pilot Project (FPP), \\ Porcon Farm, Cajamarca
}

Jorge Merino Coral ${ }^{1}$, Carlos Chuquicaja Segura ${ }^{2}$ y Ulises Pajares Gallardo ${ }^{3}$

\begin{abstract}
Resumen
En el presente trabajo se estimó el valor de uso directo del suelo en Porcón-Cajamarca, mediante el método de valor potencial para tres tasas guías de interés; teniendo en cuenta como primera alternativa, el uso forestal mediante el establecimiento de plantaciones de Pinus patula con fines comerciales, para lo cual se analizaron cuatro casos de producción de la plantación; y como segunda alternativa, el uso tradicional de ganadería extensiva de ovinos Corriedale con fines de lana y carne. Los resultados obtenidos para las tasas guías de interés de $8 \%$ y $10 \%$, indican que el mejor uso alternativo del suelo en la zona de estudio y para los casos analizados, es establecer plantaciones de pino; mientras que, para la tasa guía de interés de $12 \%$, la alternativa del uso forestal será mejor que la del uso tradicional ganadero, sólo si se incluye en la producción de la plantación a la madera rolliza, los hongos comestibles, el ecoturismo y el secuestro de carbono.
\end{abstract}

Palabras clave: valor potencial del suelo; valor de uso directo; Pinus patula; Corriedale.

\begin{abstract}
In this paper the value of direct soil use is estimated in Porcón-Cajamarca, by the method of potential value for three rates guides of interest; considering as a first alternative, forestry use by establishing plantations of Pinus patula for commercial purposes, for which four cases of production of the plantation were analyzed; and as second alternative, the traditional use of grazing Corriedale sheep with purposes of wool and meat. The results obtained for the rates guides of interest of $8 \%$ and $10 \%$, indicate than best alternative soil use in the study area and for cases analyzed, is to establish pine plantations; whereas, with an interest rate of $12 \%$, the forestry use will be better than the traditional livestock use, only if included in production the plantation to the round wood, edible mushrooms, ecotourism and carbon sequestration.
\end{abstract}

Key words: soil potential value; direct use value; Pinus patula; Corriedale.

\footnotetext{
${ }^{1}$ Consultor independiente, Perú. E-mail: lizandromc27@gmail.com

${ }^{2}$ Facultad de Ciencias Forestales, Universidad Nacional Agraria La Molina, Perú.

${ }^{3}$ Director de Investigación, Asociación Civil para la Investigación y Desarrollo Forestal (ADEFOR), Perú.
} 


\section{Introducción}

El Perú posee una extensión de 10,5 millones de hectáreas de tierras con aptitud para el establecimiento de plantaciones forestales, de las cuales 7,5 millones se encuentran en la sierra; 2,5 millones en la selva y 0,5 millones en la costa (FAO 2004).

En la región sierra, el departamento de Cajamarca destaca con una de las experiencias más importantes en forestación a nivel del país: El Proyecto Piloto de Forestación (PPF), cuyas plantaciones de distintas especies de pinos, fueron establecidas en el predio Granja Porcón entre 1983 y 1989 con el apoyo económico de la Comunidad Económica Europea y de la Cooperación Técnica Belga en convenio con el Estado peruano. A partir de 1990, el manejo de las plantaciones del PPF está a cargo de la Asociación Civil para la Investigación y Desarrollo Forestal (ADEFOR) y de la Cooperativa Agraria de Trabajadores "Atahualpa-Jerusalén" (CAT), esta última, dueña del predio (ADEFOR 2005). Actualmente, el PPF cuenta con 2389 hectáreas de área maderera, las cuales son de gran importancia para la economía de la zona y del país, considerando que para el año 2012 la producción de madera rolliza de pino, alcanzó los 73 $780 \mathrm{~m}^{3}(\mathrm{r})$ (MINAG 2013), lo cual da cuenta de la demanda que existe por esta madera. Sin embargo, hasta la fecha en el ámbito del PPF no se cuenta con información base acerca del valor económico de estas plantaciones a nivel de sus componentes suelo y vuelo, debido a que no se han realizado estudios al respecto; por tanto, se entiende que las instituciones a cargo desconocen cuál es el valor de uso directo de estos recursos forestales.

En Colombia para plantaciones de Pinus patula, ubicadas en distintas zonas de la Región Andina, se estimaron rendimientos para la especie que están entre $142 \mathrm{~m}^{3}(\mathrm{r})$ y $410 \mathrm{~m}^{3}(\mathrm{r})$ por hectárea; también, se determinaron turnos financieros óptimos que varían de 12 a 14 años y al considerar una tasa guía de interés de 6,81\%, se encontró que el valor del suelo, varía desde US\$ - 1404 a US\$ 1885 por hectárea (Restrepo et al. 2012).
En Colombia, CONIF (2013) realizó una evaluación de costos del establecimiento en plantaciones de Pinus spp; ; para lo cual, obtuvo información de distintas regiones de Colombia, así como de otros países de Sudamérica, y determinó que, establecer una plantación de la especie Pinus patula cuesta en promedio US\$ 1014 por hectárea.

En Perú, el INIA (2014) en base a sus investigaciones realizadas tanto en plantaciones de pinos como en sistemas agroforestales ubicados en las regiones de Pasco y Junín, ha calculado que establecer una plantación de pino cuesta US\$ 840 por hectárea, costo que cubre la limpieza del terreno, la instalación, los cuidados de los árboles y la mano de obra.

Fernández et al. (2012), en Argentina encontró que en plantaciones de Pinus spp., los gastos anuales de mantenimiento ascienden a US\$ 15,58 por hectárea al año, lo cual incluye el costo anual de prevención de incendios, un 3\% de los gastos anuales por administración y un $10 \%$ por concepto de imprevistos. Por su parte Novoa (2005), determinó que en Chile para plantaciones experimentales con regímenes silviculturales intensivos, los gastos anuales de mantenimiento ascienden a US\$ 20 por hectárea al año.

Referirse al recurso suelo forestal, es referirse a los suelos de aptitud forestal en general, por tanto, se incluye a los suelos sin cubierta forestal y a los suelos con cubierta forestal (bosques naturales). En el primer caso, la valoración de un suelo sin cubierta vegetal, se hace considerando su mejor uso alternativo para lo cual se proyectan los costos e ingresos a fin de determinar la renta periódica que se obtendrá; lo cual debe ser considerado a perpetuidad. La actualización de dicha renta corresponde al valor potencial del suelo; que representa el monto máximo que se podría pagar por el suelo; así mismo el valor potencial del suelo, es el aporte del suelo como factor de producción hacia la producción (Klemperer 2003).

El valor potencial del suelo, se obtiene estimando el valor actual neto de todos los futuros ingresos que un bosque o una parte de él puede 
producir; como se trata del futuro, hay que hacer estimaciones, $\mathrm{y}$ a veces por periodos prolongados; también hay que seleccionar una tasa de interés que refleje la importancia del tiempo para llevar a cabo las capitalizaciones que son parte de los cálculos. La gran ventaja de este método es que se toma en cuenta los futuros ingresos y costos, básicamente son éstos los que determinan el valor de una propiedad, si su uso es continuo como es el caso de un bosque manejado (Petriceks 1981).

La producción forestal con rodales coetáneos comienza con el establecimiento del rodal mediante regeneración natural o plantaciones, siguen varias intervenciones silviculturales, como limpias, entresacas, y al terminarse el turno, la explotación final. En un bosque manejado, este proceso se puede repetir tantas veces como se desee, incluso a perpetuidad; en términos de valoración se trata de rentas periódicas infinitas, siendo el periodo la duración del turno. Para poder calcular la renta neta, hay que llevar todos los costos de manejo y los ingresos obtenidos al último año del turno (capitalizar), y ahí realizar la suma de todos los ingresos y restarle a ésta la suma de todos los costos; al actualizar esta renta neta, se obtendrá el valor del suelo (Vs), aplicando la fórmula general, conocida como fórmula de Faustmann (Petriceks 1981):

$$
\begin{gathered}
\mathrm{Vs}=\left[\mathrm{I}+\mathrm{Ea}(1,0 \mathrm{p})^{\mathrm{n}-\mathrm{a}}+\mathrm{Eb}(1,0 \mathrm{p})^{\mathrm{n}-\mathrm{b}}+\left(\mathrm{r}\left(1,0 \mathrm{p}^{\mathrm{n}}-1\right)\right) / 0,0 \mathrm{p}-\mathrm{P}(1,0 \mathrm{p})^{\mathrm{n}}-\right. \\
\left.\mathrm{Cd}(1,0 \mathrm{p})^{\mathrm{n}-\mathrm{d}}-\left(\mathrm{c}\left(1,0 \mathrm{p}^{\mathrm{n}}-1\right)\right) / 0,01 \mathrm{p}\right] / 1,0 \mathrm{pn}-1
\end{gathered}
$$

Donde:

I = ingreso neto de la explotación final

$\mathrm{Ea}=$ ingreso de la entresaca en el año a

$\mathrm{Eb}=$ ingreso de la entresaca en el año $\mathrm{b}$

$r=$ ingreso anual, durante todo el turno

$\mathrm{P}=$ costo de establecimiento (plantación)

$\mathrm{Cd}=$ costo de intervención en el año d

$\mathrm{c}=$ costo anual de administración y mantenimiento de la plantación

$\mathrm{n}=$ duración del turno

$\mathrm{p}=$ tasa guía de interés
La tasa guía de interés o simplemente tasa de interés, es un costo de oportunidad, ya que puede basarse en las oportunidades de inversión de la empresa o persona que desee desarrollar un proyecto forestal. Respecto a la relación entre esta tasa y el valor del suelo, Prieto et al. (1998) y Ortuño et al. (2007) señalan que debido a lo prolongados que son los turnos de producción en los proyectos forestales, el valor del suelo es muy sensible al tipo de interés utilizado; cumpliéndose que a mayor tasa de interés menor será el valor del suelo.

En la presente investigación se determina el valor de uso directo del suelo en el área del Proyecto Piloto de Forestación (PPF), teniendo en cuenta el uso forestal y el uso tradicional ganadero. Los resultados obtenidos, permitirán un mejor entendimiento del recurso suelo como factor de producción y contribuirán a tomar mejores decisiones en la definición de uso de los suelos forestales en el ámbito del estudio.

\section{Materiales y Métodos}

\section{Zona de estudio}

El predio Granja Porcón, se ubica a 30 kilómetros al norte de la ciudad de Cajamarca, entre las provincias de Cajamarca y San Pablo en la Región Cajamarca; geográficamente se sitúa a $07^{\circ} 02^{\prime}$ de latitud Sur y $78^{\circ} 38^{\prime}$ de longitud Oeste; entre los 2900 a $3800 \mathrm{msnm}$. La precipitación promedio anual es de $1127 \mathrm{~mm}$; en general el predio presenta un relieve variado, el rango de pendiente es de 30\% a 70\%. Los suelos se caracterizan por ser superficiales a moderadamente profundos, formados por derivados de rocas volcánicas, son de textura media, con un perfil dominante $\mathrm{A} / \mathrm{C}$; presentan un $\mathrm{pH}$ de 4,5 a 5,2 (suelos ácidos); de acuerdo a estas características se clasifican como Andosoles y su aptitud se orienta principalmente para la producción forestal y para el pastoreo controlado.

El estudio se hizo en base a la información contable y registros de producción de ADEFOR; estudio de Caso Granja Porcón, elaborado por ADEFOR; primer Compendio Es- 
tadístico Agrario (1950-1991) del Ministerio de Agricultura; Plan General de Manejo Forestal de las plantaciones del Proyecto Piloto de Forestación (PPF), elaborado por ADEFOR; trabajos de investigación referentes a las plantaciones forestales de Granja Porcón; estudios técnicos, económicos y turísticos, realizados a nivel de Cajamarca y del material audiovisual disponible en ADEFOR.

\section{Metodología para valorar el suelo con uso forestal}

Tomando como fuentes de información el Plan General de Manejo Forestal de las plantaciones del PPF elaborado por ADEFOR (2005) y el estudio de Carton (2000) donde se da a conocer los resultados del test de adaptación de especies de las plantaciones forestales demostrativas establecidas en Granja Porcón, se consideró lo siguiente: la especie Pinus patula es la que mejor se ha adaptado y por tanto la seleccionada con fines de establecimiento y reposición en Granja Porcón; el turno de la rotación para alcanzar la cosecha final es de 30 años y los turnos se repetirán a perpetuidad.

\section{Selección del área de estudio}

Se seleccionó un área para el análisis económico, teniendo en cuenta año de establecimiento del rodal. El área seleccionada fue establecida el año 1983 y por tanto al año 2013 ya cumplió el turno predefinido de 30 años. Esta área la conforman las unidades de manejo denominadas como: Pululo y Campanario, las cuales albergan una plantación de Pinus patula, de la que se ha extraído madera (raleo comercial), hongos comestibles, y además, al igual que todo el territorio de la granja, brinda servicios de recreación y turismo. Hasta la fecha de recopilación de información para el presente estudio, aún no se ha realizado la cosecha final.

\section{Procesamiento y organización de la información}

Se determinaron los costos e ingresos por actividad y por periodo generados durante el manejo de la plantación forestal de la especie Pinus patula desde su establecimiento en 1983 (0 años) hasta el 2013 (30 años), teniendo en cuenta la producción de bienes y servicios:

\section{Producción de madera rolliza (1983 - 2013)}

Se obtuvo el costo de establecimiento de la plantación forestal a partir de los datos registrados por la empresa VIPLANTEX en la evaluación económica que realizó de las plantaciones forestales de Granja Porcón y Huacraruco (ADEFOR 1998).

Luego, en base a la información de los archivos contables de ADEFOR, se calculó el costo total del raleo comercial ejecutado el año 2005 en base a los siguientes rubros: (a) operaciones, (b) mano de obra, (c) otros costos y (d) costos fijos. Seguidamente, se calculó el ingreso total que se obtuvo por la venta de la madera rolliza producida en dicho raleo; a partir de los cuales, se determinó el ingreso neto por raleo comercial.

A continuación, se calculó el costo de la poda, la cual se ejecutó el año 2005 después del raleo y seguidamente se estimó el gasto anual de mantenimiento de la plantación, el cual se considera un gasto constante que se ha realizado cada año desde que fue establecida la plantación hasta la fecha en que se ejecutó el presente estudio.

Por último, en base al volumen promedio de madera en pie, aprovechable, existente por hectárea de la especie Pinus patula según el inventario forestal realizado por ADEFOR el año 2013 y al valor de la madera en pie promedio, se proyectó el ingreso neto por cosecha final.

\section{Producción de hongos comestibles deshidratados (2004 - 2013)}

Se obtuvo la producción promedio de hongos comestibles de la especie Suillus luteus (frescos o recién recolectados) y porcentaje de materia seca para esta especie $(9,48 \%)$, a partir de los resultados de García (1999) y se calculó la producción promedio de hongos comestibles deshidratados. A continuación, en base a la información proporcionada por el área de investigación de ADEFOR, se estimó la proporción del área que ocupan los hongos en una hectárea de pino (25\%); con este dato se calculó el rendimiento bruto en la producción de hongos comestibles deshidratados. 
Finalmente, se calculó el ingreso neto anual por la venta de hongos comestibles deshidratados; para lo cual se utilizó el rendimiento bruto, el costo de producción unitario y el precio de venta unitario, estos dos últimos datos fueron extraídos de Fundación Romero (2009).

\section{Ecoturismo (2002 - 2013)}

Se obtuvo datos históricos de la cantidad de turistas nacionales y extranjeros que llegan a Cajamarca y visitan la Granja Porcón, a partir de DIRCETUR (2006), CAT (2009) y MINCETUR (2013); con estos datos se determinó el número promedio de turistas que acuden a la granja anualmente. Luego, se estimaron los costos administrativos y operativos que se generan anualmente por el desarrollo de las actividades ecoturísticas en Granja Porcón y con la sumatoria de éstos, se calculó el costo total anual por concepto de ecoturismo. Seguidamente, con el número de turistas promedio anual y teniendo en cuenta el monto promedio que se cobra por ingresar a Granja Porcón, se calculó el ingreso total anual por concepto de ecoturismo. Con los datos del costo e ingreso total anual y considerando que el área destinada para la recreación de los turistas abarca 5749 hectáreas del total de hectáreas de pino que se han establecido en Granja Porcón, se calculó el ingreso neto anual por ecoturismo.

\section{Secuestro de carbono (1983 - 2013)}

Se obtuvo la cantidad promedio de toneladas de carbono que anualmente secuestra una hectárea de pino, a partir de los resultados de Alegre et al. (2004) y este dato se convirtió a toneladas de dióxido de carbono equivalente $\left(\mathrm{CO}_{2} \mathrm{e}\right)$. Seguidamente, en base a la información de un estudio elaborado por Pro Chile (2012), se obtuvo el precio promedio de una tonelada de dióxido de carbono equivalente $\left(\mathrm{CO}_{2} \mathrm{e}\right)$, la cual es la cantidad equivalente a un bono de carbono que se comercializa a través del mercado voluntario de bonos de carbono; así mismo, se estimó el costo por tonelada secuestrada de $\mathrm{CO}_{2}$ e, a partir de los resultados de Chidiak et al. (2003). Finalmente, se calculó el ingreso neto anual por secuestro de carbono.

\section{Estimación del valor de uso directo del suelo}

Una vez determinados los costos e ingresos, se procedió a calcular el valor de uso directo del suelo para tasas guías de interés de $8 \% ; 10 \%$ y $12 \%$, aplicando la fórmula de Faustmann, para los siguientes casos:

Caso 1: Considerando sólo la producción de madera rolliza en pie.

$$
V s=\frac{Y_{r}+T_{22}(1+i)^{n-22}-C(1+i)^{n}-L_{22}(1+i)^{n-22}-e\left\{(1+i)^{n}-1\right\} / i}{(1+i)^{n}-1}
$$

Caso 2: Considerando la producción de madera rolliza en pie y hongos comestibles.

$$
\begin{aligned}
V s= & Y_{r}+T_{22}(1+i)^{n-22}+F_{2\{}\left\{(1+i)^{n-20}-1\right\} i-C(1+i)^{n}-L_{22}(1+i)^{n-22}- \\
& e\left\{(1+i)^{n}-1\right\} /[i] /\left[(1+i)^{n}-1\right]
\end{aligned}
$$

Caso 3: Considerando la producción de madera rolliza en pie, hongos comestibles y ecoturismo.

$\mathrm{Vs}=$

$$
\left[Y_{r}+T_{22}(1+i)^{n-22}+F_{21}\left\{(1+i)^{n-20}-1\right\} i+S_{19}\left\{(1+i)^{n-18}-1\right\} i-\right.
$$

$C(1+i)^{n}-L_{22}(1+i)^{n-22}-e\left\{(1+i)^{n}-1\right\} i\left[j /\left[(1+i)^{n}-1\right]\right.$

Caso 4: Considerando la producción de madera rolliza en pie, hongos comestibles, ecoturismo y secuestro de carbono.

$V S=\left[Y_{r}+T_{22}(1+i)^{n-22}+F_{21}\left\{(1+i)^{n-20}-1\right\} / i+S_{19}\left\{(1+i)^{n-18}-1\right\} / i+\right.$ $\left.K\left\{(1+i)^{n}-1\right\} / i-C(1+i)^{n}-L_{22}(1+i)^{n-22}-e\left\{(1+i)^{n}-1\right\} / i\right] /\left[(1+i)^{n}-1\right]$

Donde:

Vs: valor potencial del suelo (US\$/ha)

Yr: ingreso neto por cosecha final (US\$/ha)

$\mathrm{T}_{22}$ : ingreso neto por raleo comercial en el año 22 (US\$/ha)

$\mathrm{F}_{21}$ : ingreso neto anual por hongos comestibles deshidratados del año 21 al año 30 (US\$/ha/año)

$\mathrm{S}_{19}$ : ingreso neto anual por ecoturismo del año $19 \mathrm{al}$ año 30 (US\$/ha/año)

$\mathrm{K}$ : ingreso neto anual por secuestro de carbono (US\$/ ha/año)

C: costo de establecimiento de la plantación (US\$/ha)

$\mathrm{L}_{22}$ : costo de la poda realizada en el año 22 (US\$/ha)

e: gastos anuales de mantenimiento (US\$/ha/año)

$\mathrm{n}$ : número de años de la rotación ( $\mathrm{n}=30$ años)

i: tasa guía de interés (TGI). 


\section{Metodología para valorar el suelo con uso tradicional ganadero}

Tomando como fuentes de información el Estudio de Caso Granja Porcón elaborado por ADEFOR (1993) y el estudio de Carton (2000), en las cuales se describe el uso que antiguamente se le daba a las áreas del PPF, se consideró lo siguiente: el ganado ovino que tradicionalmente se ha criado en Granja Porcón es de la raza Corriedale, la cual es una raza de doble propósito para producción de lana y carne; el ciclo productivo es anual y la crianza de ovinos se mantendrá a perpetuidad.

\section{Procesamiento y organización de la infor- mación}

Se determinaron los costos e ingresos generados durante la crianza de ganado ovino de raza Corriedale bajo un sistema extensivo, considerando el año 1983 como el inicio de los ciclos productivos anuales y en base a los siguientes rubros:

Costo del capital: se calculó el costo de capital en base a la inversión total estimada y el cálculo se realizó para tasas guías de interés de $8 \%$; $10 \%$ y $12 \%$. Mano de obra: se obtuvo el número de jornales por día, empleados en las labores de pastoreo y el periodo de trabajo que se dedica a esta actividad, a partir de ADEFOR (1993). Seguidamente en base a datos históricos del MINAG (1992) y al tipo de cambio de la moneda vigente en el año 1983, se calculó el costo del jornal. Por último, con estos datos se calculó el costo total de la mano de obra. Sanidad animal: en base al número de cabezas de ganado ovino y a la equivalencia de una cabeza de ganado en unidades ovinas, se calculó el número de unidades ovinas promedio anual. Seguidamente se obtuvo el costo de las dosificaciones aplicadas a los ovinos, a partir de ADEFOR (1993). Finalmente, con estos datos se calculó el costo total por sanidad animal. Depreciación de la infraestructura y costos administrativos: la depreciación de la infraestructura en uso para la crianza y manejo del ganado ovino y los costos administrativos que se generan durante el proceso productivo, se obtuvieron directamente de ADEFOR (1993). Área total de pastoreo: en base al dato promedio de capacidad de carga animal según ADEFOR (1993) y al número de unidades ovinas promedio anual, se estimó el área total que ocupa el ganado ovino para su pastoreo. Costo de producción por hectárea: con la sumatoria de los rubros anteriores se determinó el costo total de producción y considerando el área total de pastoreo, se procedió a calcular el costo de producción por hectárea. Ingreso por la venta de lana de ovino: en base a datos históricos del precio al productor de lana de ovino según Villanueva (1994) y al tipo de cambio de la moneda vigente en el año 1983, se calculó el precio de venta del kilogramo de lana. Seguidamente en base a la producción promedio de lana por unidad ovina extraída de ADEFOR (1993) y a la capacidad de carga animal, se calculó el rendimiento por hectárea. Por último, con estos datos se calculó el ingreso por la venta de lana de ovino. Ingreso por la venta de carne de ovino: en base a datos históricos del precio de la carne de ovino en centros de producción según el MINAG (1992) y al tipo de cambio de la moneda vigente en el año 1983, se calculó el precio de venta del kilogramo de carne. Seguidamente en base a la cantidad de kilogramos de carne que se obtiene en promedio de una unidad ovina y a la capacidad de carga animal, se calculó el rendimiento por hectárea. Finalmente, con estos datos se calculó el ingreso por la venta de carne de ovino. Ingreso total anual por hectárea: con la sumatoria de los ingresos por la venta de lana y carne calculados en los dos puntos anteriores, se calculó el ingreso total anual por hectárea.

\section{Estimación del valor de uso directo del suelo}

Por la diferencia entre el ingreso total por hectárea y el costo de producción por hectárea se calculó la renta anual y con ésta se procedió a calcular el valor de uso directo del suelo para tasas guías de interés de (TGI) $8 \% ; 10 \%$ y $12 \%$, empleando la relación de valor actualizado de una serie anual perpetua:

$$
\mathrm{Vs}=\mathrm{R} / \mathrm{i}
$$

Donde: Vs: valor potencial del suelo (US\$/ha); $\mathrm{R}$ : renta anual (US\$/ha/año) e i: tasa guía de interés. 


\section{Resultados}

\section{Uso forestal}

En el Cuadro 1, los resultados muestran que en 1983 se hizo la plantación, cuyo costo de establecimiento está en base a una densidad de 1300 plantas por hectárea (ADEFOR 1998); se observa también, que a la edad de 22 años se hizo la única poda a la plantación durante el turno y a lo largo del mismo, se han realizado gastos anuales de mantenimiento que incluyen costos por administración y costos por mantenimiento de caminos.

En el Cuadro 2, se muestran el ingreso neto por cosecha final que ha sido estimado para la edad de 30 años; el ingreso neto por raleo comercial se dio a los 22 años y éste es el único que se ha realizado durante el turno; asimismo, se observa que en los últimos 10 año del turno, se han obtenido ingresos netos anuales por la venta de hongos comestibles deshidratados, que son hongos que fructifican en el suelo de la plantación; también, en los últimos 12 años del turno, se han obtenido ingresos netos anuales por el servicio de ecoturismo desarrollado en el área de la plantación; y además, se ha estimado un ingreso neto anual por secuestro de carbono; pero es importante resaltar que actualmente este ingreso, no se percibe; sin embargo, por la importancia ambiental, económica y social que tiene este servicio ambiental, se decidió incluirlo en el análisis económico.

En el Cuadro 3, se muestran los costos capitalizados hacia el último año del turno (ubica-

\begin{tabular}{|c|c|c|c|c|}
\hline $\begin{array}{c}\text { Año y/o } \\
\text { período }\end{array}$ & $\begin{array}{c}\text { Edad de } \\
\text { plantación } \\
\text { (años) }\end{array}$ & Ítem & Unidades & Monto \\
\hline 1983 & 0 & $\begin{array}{c}\text { Costo de esta- } \\
\text { blecimiento }\end{array}$ & US\$/ha & 473,71 \\
\hline 2005 & 22 & $\begin{array}{c}\text { Costo de } \\
\text { poda }\end{array}$ & US\$/ha & 4,10 \\
\hline $1983-2013$ & $0-30$ & $\begin{array}{c}\text { Gastos } \\
\text { anuales de } \\
\text { manten- } \\
\text { imiento }\end{array}$ & US\$/ha/año & 2,69 \\
\hline
\end{tabular}

Cuadro 1. Costos generados durante el turno forestal. dos a la edad de 30 años) con las tres tasas guías de interés $(8 \% ; 10 \%$ y $12 \%)$ que se han considerado en el presente estudio; asimismo, se observan los montos totales en cada tasa; cabe resaltar que esta capitalización según lo describe Petriceks (1981), es parte de los cálculos que se realizan al aplicar la fórmula de Faustmann. Al aplicar el interés compuesto, se observa que los valores capitalizados de los costos se incrementan al aumentar la tasa guía de interés. El costo de establecimiento tiene una gran incidencia en los costos capitalizados, representa más del $90 \%$ del costo total.

En el Cuadro 4, se muestran los ingresos obtenidos por la producción de bienes y servicios capitalizados hacia el último año del turno (ubicados a la edad de 30 años) con las tres tasas guías de interés $(8 \% ; 10 \%$ y $12 \%)$ que se han considerado en el presente estudio; asimismo, se observan los montos totales en cada tasa y para cada uno de los casos evaluados.

En el Cuadro 5, se muestra el valor de uso directo del suelo para los cuatro casos evaluados, en el uso forestal. Para una tasa guía de interés de $8 \%$, el valor del suelo considerando sólo la producción de madera rolliza en pie es de 1 116,97 US\$/ha; cuando se considera, además, la producción de hongos comestibles el valor del suelo se incrementa a 1220,52 US\$/ha, que representa un incremento del valor del suelo en $9 \%$ en relación al caso anterior (Caso 1). Cuando se considera la producción de madera rolliza, hongos comestibles y ecoturismo el valor del suelo es 1244,75 US $\$$ /ha; en este caso el valor del suelo se incrementa en $2 \%$ con respecto al caso anterior (Caso 2). Y al considerar la producción de madera rolliza, hongos comestibles, ecoturismo y secuestro de carbono el valor del suelo alcanza un valor de 2 701,62 US\$/ha; que representa un incremento de $117 \%$ con respecto al caso anterior (Caso 3).

Para las TGI de $10 \%$ y $12 \%$, se observa una tendencia similar de incremento del valor del suelo en la medida que se incluyan nuevos productos del bosque, en los cuatro casos.

En el Cuadro 5, también se puede observar que el valor del suelo, para cada caso, disminuye 


\begin{tabular}{|c|c|c|c|c|}
\hline $\begin{array}{l}\text { Año } y / o \\
\text { período }\end{array}$ & $\begin{array}{c}\text { Edad de } \\
\text { plantación } \\
\text { (años) }\end{array}$ & Ítem & Unidades & Monto \\
\hline & & Madera rolliza & & \\
\hline 2013 & 30 & Ingreso neto por cosecha final & US\$/ha & 14774,02 \\
\hline 2005 & 22 & Ingreso neto por raleo comercial & US\$/ha & 231,12 \\
\hline & & Otros ingresos & & \\
\hline $2004-2013$ & $21-30$ & $\begin{array}{l}\text { Ingreso neto anual por hongos } \\
\text { comestibles deshidratados }\end{array}$ & US\$/ha/año & 64,78 \\
\hline $2002-2013$ & $19-30$ & Ingreso neto anual por ecoturismo & US\$/ha/año & 11,57 \\
\hline $1983-2013$ & $0-30$ & $\begin{array}{l}\text { Ingreso neto anual por secuestro de } \\
\text { carbono }\left(\mathrm{CO}_{2} \mathrm{e}\right)\end{array}$ & US\$/ha/año & 116,55 \\
\hline
\end{tabular}

Cuadro 2. Ingresos generados durante el turno forestal.

\begin{tabular}{|c|l|l|r|r|r|}
\hline \multirow{2}{*}{$\begin{array}{c}\text { Edad } \\
\text { (años) }\end{array}$} & \multicolumn{1}{|c|}{ Ítem } & \multirow{2}{*}{ Unidades } & \multicolumn{3}{|c|}{ Tasa guía de interés } \\
\cline { 4 - 6 } & & & $\mathbf{8 \%}$ & $\mathbf{1 0} \%$ & \multicolumn{1}{|c|}{$\mathbf{1 2 \%}$} \\
\hline 30 & $\begin{array}{l}\text { Costo de establec- } \\
\text { imiento }\end{array}$ & US\$/ha & 4766,78 & 8265,96 & 14192,31 \\
\hline 30 & Costo de poda & US\$/ha & 7,59 & 8,79 & 10,15 \\
\hline 30 & $\begin{array}{l}\text { Gastos anuales de } \\
\text { mantenimiento }\end{array}$ & US\$/ha & 304,73 & 442,49 & 649,18 \\
\hline
\end{tabular}

Cuadro 3. Costos capitalizados hacia el último año del turno.

\begin{tabular}{|c|c|c|c|c|c|}
\hline \multirow{2}{*}{$\begin{array}{l}\text { Edad } \\
\text { (años) }\end{array}$} & \multirow{2}{*}{ Ítem } & \multirow{2}{*}{ Unidades } & \multicolumn{3}{|c|}{ Tasa guía de interés } \\
\hline & & & $8 \%$ & $10 \%$ & $12 \%$ \\
\hline & Madera rolliza & & & & \\
\hline 30 & Ingreso neto por cosecha final & US\$/ha & 14774,02 & 14774,02 & 14774,02 \\
\hline \multirow[t]{2}{*}{30} & Ingreso neto por raleo comercial & US\$/ha & 427,79 & 495,43 & 572,24 \\
\hline & Otros ingresos & & & & \\
\hline 30 & $\begin{array}{l}\text { Ingreso neto anual por hongos } \\
\text { comestibles deshidratados }\end{array}$ & US\$/ha & 938,44 & 1032,43 & 1136,81 \\
\hline 30 & $\begin{array}{l}\text { Ingreso neto anual por eco- } \\
\text { turismo }\end{array}$ & US\$/ha & 219,57 & 247,42 & 279,22 \\
\hline 30 & $\begin{array}{l}\text { Ingreso neto anual por secuestro } \\
\text { de carbono }\left(\mathrm{CO}_{2} \mathrm{e}\right)\end{array}$ & US\$/ha & 13203,16 & 19171,78 & 28127,32 \\
\hline & TOTAL (Caso 1) & US\$/ha & 15201,81 & 15269,45 & 15346,26 \\
\hline & TOTAL (Caso 2) & US\$/ha & 16140,25 & 16301,87 & 16483,07 \\
\hline & TOTAL (Caso 3) & US\$/ha & 16359,81 & 16549,29 & 16762,29 \\
\hline & TOTAL (Caso 4) & US\$/ha & 29562,97 & 35721,07 & 44889,62 \\
\hline
\end{tabular}

Cuadro 4. Ingresos capitalizados hacia el último año del turno 


\begin{tabular}{|c|c|c|c|c|}
\hline \multicolumn{5}{|c|}{$\begin{array}{l}\text { Uso forestal } \\
\text { (Plantaciones de Pinus patula) }\end{array}$} \\
\hline \multirow{2}{*}{ Caso } & \multirow{2}{*}{ Producto } & \multicolumn{3}{|c|}{$\begin{array}{r}\text { Valor de uso direccto del } \\
\text { suelo (US\$/ha) }\end{array}$} \\
\cline { 3 - 5 } & & \multicolumn{2}{c|}{ Tasa guía de interés (TGI) } \\
\cline { 3 - 5 } & & $\mathbf{8 \%}$ & $\mathbf{1 0 \%}$ & $\mathbf{1 2 \%}$ \\
\hline 2 & Madera rolliza & 1116,97 & 398,33 & 17,08 \\
\hline \multirow{2}{*}{3} & $\begin{array}{l}\text { Madera rolliza y } \\
\text { hongos comes- } \\
\text { tibles }\end{array}$ & 1220,52 & 461,09 & 56,33 \\
& $\begin{array}{l}\text { Madera rolliza, } \\
\text { hongos comesti- } \\
\text { bles y ecoturismo }\end{array}$ & 1244,75 & 476,13 & 65,98 \\
\hline \multirow{2}{*}{4} & $\begin{array}{l}\text { Madera rolliza, } \\
\text { hongos comesti- } \\
\text { bles, ecoturismo } \\
\text { y secuestro de } \\
\text { carbono }\end{array}$ & 2701,62 & 1641,63 & 1037,23 \\
\hline
\end{tabular}

Cuadro 5. Valor de uso directo del suelo con uso forestal.

al aumentar la TGI. Cuando se considera como producto solo la madera rolliza en pie y una TGI de 8\%, el valor del suelo es 1116,97 US\$/ ha; mientras que con una TGI de $12 \%$ el valor del suelo se reduce a 17,08 US\$/ha. Para el caso que se considera como productos a la madera en pie, hongos comestibles, servicio turístico y servicio de secuestro de carbono, para una TGI de $8 \%$, el valor del suelo es 2 701,62 US\$/ha; mientras que con una TGI de $12 \%$, el valor del suelo se reduce a 1037,23 US\$/ha.

\section{Uso tradicional ganadero}

En el Cuadro 6, se muestran los montos promedios anuales de todos los rubros que intervienen en la crianza de ganado ovino; observándose que de todos los rubros, el único que varía según la tasa guía de interés es el costo del capital, ya que a medida que la tasa guía de interés disminuye el costo del capital también disminuye; por tanto, la variación en el costo de producción por hectárea, depende únicamente del costo del capital.

En el Cuadro 7, se muestran los rendimientos por hectárea de carne y lana; así como el ingreso promedio anual por hectárea, obtenido por la venta de ambos productos. Observándose que el ingreso por el concepto de carne es mayor que el de lana; el ingreso promedio por concepto de carne representa el 94\% del ingreso total anual por hectárea.

En el Cuadro 8, se muestra el valor de la renta anual, calculado por la diferencia entre el ingreso total y el costo de producción para las tres TGI que se han considerado ( $8 \% ; 10 \%$ y $12 \%)$. Observándose que la renta anual disminuye ligeramente al aumentar la TGI.

En el Cuadro 9, se muestra el valor de uso directo del suelo, bajo el uso tradicional ganadero. Estos resultados, al igual que en el uso forestal, disminuyen a medida que la tasa guía de interés aumenta; para una TGI de $8 \%$ el valor del suelo es 474,25 US\$/ha; para una

\begin{tabular}{|c|c|c|c|c|c|c|c|c|}
\hline \multicolumn{2}{|c|}{$\begin{array}{c}\text { Costo del capital } \\
\text { (US\$/año) }\end{array}$} & \multirow{2}{*}{$\begin{array}{c}\begin{array}{c}\text { Mano } \\
\text { de obra }\end{array} \\
\text { (US\$/año) }\end{array}$} & \multirow{2}{*}{$\begin{array}{c}\begin{array}{c}\text { Sanidad } \\
\text { animal }\end{array} \\
\text { (US\$/año) }\end{array}$} & \multirow{2}{*}{$\begin{array}{r}\begin{array}{c}\text { Depreciación } \\
\text { de infraestruc- } \\
\text { tura (US\$/año) }\end{array} \\
7457,86\end{array}$} & \multirow{2}{*}{$\begin{array}{c}\begin{array}{c}\text { Costos } \\
\text { adminis- } \\
\text { trativos } \\
\text { (US\$/año) }\end{array} \\
4162,88\end{array}$} & \multirow{2}{*}{$\begin{array}{c}\begin{array}{c}\text { Costo total de } \\
\text { producción } \\
\text { (US\$/año) }\end{array} \\
71664,34\end{array}$} & \multirow{2}{*}{$\begin{array}{c}\begin{array}{c}\text { Área } \\
\text { total de } \\
\text { pastoreo } \\
\text { (ha) }\end{array} \\
6057\end{array}$} & \multirow{2}{*}{$\begin{array}{c}\begin{array}{c}\text { Costo de } \\
\text { producción }\end{array} \\
\text { por hectárea } \\
\text { (US\$/ha/año) } \\
11,83\end{array}$} \\
\hline $\begin{array}{l}\mathrm{TGI}^{1} \\
12 \%\end{array}$ & 42768,00 & & & & & & & \\
\hline TGI & \multirow{2}{*}{35640,00} & \multirow{2}{*}{8190,60} & \multirow{2}{*}{9085,00} & \multirow{2}{*}{7457,86} & \multirow{2}{*}{4162,88} & \multirow{2}{*}{64536,34} & \multirow{2}{*}{6057} & \multirow{2}{*}{10,65} \\
\hline $10 \%$ & & & & & & & & \\
\hline TGI & \multirow{2}{*}{28512,00} & \multirow{2}{*}{8190,60} & \multirow{2}{*}{9085,00} & \multirow{2}{*}{7457,86} & \multirow{2}{*}{4162,88} & \multirow{2}{*}{57408,34} & \multirow{2}{*}{6057} & \multirow{2}{*}{9,48} \\
\hline $8 \%$ & & & & & & & & \\
\hline
\end{tabular}

Cuadro 6. Costo de producción en la crianza de ganado ovino. Donde: ${ }^{1} \mathrm{TGl}=$ tasa guía de interés. 


\begin{tabular}{|c|c|r|r|r|}
\hline $\begin{array}{c}\text { Ciclo } \\
\text { produc- } \\
\text { tivo }\end{array}$ & Producto & $\begin{array}{c}\text { Precio de } \\
\text { venta unitario } \\
\text { (US\$/Kg) }\end{array}$ & $\begin{array}{c}\text { Rendimiento } \\
\text { por hectárea } \\
\text { (Kg/ha/año) }\end{array}$ & $\begin{array}{c}\text { Ingreso por } \\
\text { hectárea } \\
\text { (US\$/ha/año) }\end{array}$ \\
\hline \multirow{2}{*}{ Anual } & Lana & 0,66 & 4,35 & 2,87 \\
\cline { 2 - 5 } & Carne & 1,10 & 40,50 & 44,55 \\
\hline \multicolumn{4}{|c|}{ Ingreso Total Anual } \\
\hline
\end{tabular}

Cuadro 7. Ingresos generados por la venta de lana y carne de ovino.

TGI de $10 \%$ el valor del suelo es 367,70 US\$/ ha y para una TGI de $12 \%$ el valor del suelo es 296,58 US\$/ha.

\section{Análisis comparativo}

En el Cuadro 10 se presenta el valor del suelo para las dos alternativas de uso, según la tasa guía de interés correspondiente.

El Cuadro 10 muestra que, para las TGI de 8\% y $10 \%$, el valor del suelo con uso forestal es mayor que el valor del suelo con el uso tradicional de ganadería extensiva de ovinos, en los cuatro casos analizados; siendo mayor la diferencia para la TGI de $8 \%$, comparado con la de TGI de $10 \%$.

Para la TGI de $8 \%$, se observa que el valor del suelo con el uso forestal es mayor que el correspondiente valor del suelo con el uso tradicional de ganadería extensiva. Para el Caso 1, el uso forestal tiene una ventaja de $136 \%$ sobre el uso tradicional, en el Caso 2 la ventaja del uso forestal es de 157\%, en el Caso 3 la ventaja del uso forestal es de $162 \%$ y para el Caso 4 la ventaja se incrementa a $470 \%$ sobre el uso tradicional.

Para la TGI de $10 \%$, se observa que el valor

\begin{tabular}{|c|c|c|c|}
\hline TGI $^{1}$ & $\begin{array}{c}\text { Costo de } \\
\text { producción } \\
\text { por hectárea } \\
\text { (US\$/ha/año) }\end{array}$ & $\begin{array}{c}\text { Ingreso total } \\
\text { por hectárea } \\
\text { (US\$/ha/año) }\end{array}$ & $\begin{array}{c}\text { Renta anual } \\
\text { (US\$/ha/ } \\
\text { año) }\end{array}$ \\
\hline $12 \%$ & 11,83 & 47,42 & $\mathbf{3 5 , 5 9}$ \\
\hline $10 \%$ & 10,65 & 47,42 & $\mathbf{3 6 , 7 7}$ \\
\hline $8 \%$ & 9,48 & 47,42 & $\mathbf{3 7 , 9 4}$ \\
\hline
\end{tabular}

Cuadro 8. Renta anual. Donde: TGI' ${ }^{1}$ Tasa guía de interés. del suelo con el uso forestal sigue siendo mayor que el correspondiente valor del suelo con uso tradicional de ganadería extensiva; pero con una menor ventaja que el caso anterior. Observándose que, para el Caso 1, el uso forestal tiene una ventaja de solo $8 \%$ sobre el uso tradicional, en el Caso 2 la ventaja del uso forestal es de $25 \%$, en el Caso 3 la ventaja del uso forestal es de $29 \%$ y para el Caso 4 la ventaja se incrementa a $346 \%$ sobre el uso tradicional.

Para la TGI de 12\%, para los Casos 1, 2 y 3 se observa que, con el uso forestal el valor del suelo es menor que con el uso tradicional de ganadería; mientras que para el Caso 4, se mantiene la ventaja del uso forestal sobre el uso tradicional de ganadería de ovinos, en el orden de $250 \%$. Lo cual pone en evidencia que de lograrse incluir en el mercado el servicio de secuestro de carbono, el uso forestal otorgará un mayor valor al suelo que, el uso tradicional de ganadería extensiva de ovinos, para las TGI consideradas. En todos los casos y para las dos alternativas de uso consideradas, se observa que el valor del suelo disminuye, en la medida que se incrementa la TGI.

\begin{tabular}{|c|c|}
\hline $\begin{array}{c}\text { Uso tradicional ganadero } \\
\text { (Crianza extensiva de gana- } \\
\text { do ovino raza Corriedale) }\end{array}$ \\
\hline $\begin{array}{c}\text { Tasa guía de } \\
\text { interés (TGI) }\end{array}$ & $\begin{array}{c}\text { Valor de } \\
\text { uso directo } \\
\text { del suelo } \\
\text { (US\$/ha) }\end{array}$ \\
\hline $12 \%$ & 296,58 \\
\hline $10 \%$ & 367,70 \\
\hline $8 \%$ & 474,25 \\
\hline
\end{tabular}

Cuadro 9. Valor de uso directo del suelo con uso tradicional ganadero.

\begin{tabular}{|c|c|r|r|r|r|r|}
\hline \multirow{2}{*}{ Caso } & \multicolumn{2}{|c|}{$\mathbf{8 \%}$} & \multicolumn{2}{c|}{$10 \%$} & \multicolumn{2}{c|}{$11 \%$} \\
\cline { 2 - 7 } & $\begin{array}{c}\text { Uso } \\
\text { Forestal }\end{array}$ & $\begin{array}{c}\text { Uso } \\
\text { Ganadería }\end{array}$ & $\begin{array}{c}\text { Uso } \\
\text { Forestal }\end{array}$ & $\begin{array}{c}\text { Uso } \\
\text { Ganadería }\end{array}$ & $\begin{array}{c}\text { Uso } \\
\text { Forestal }\end{array}$ & $\begin{array}{c}\text { Uso } \\
\text { Ganadería }\end{array}$ \\
\hline 1 & 1116,97 & 474,25 & 398,33 & 367,70 & 17,08 & 296,58 \\
\hline 2 & 1220,52 & 474,25 & 461,09 & 367,70 & 56,33 & 296,58 \\
\hline 3 & 1244,75 & 474,25 & 476,13 & 367,70 & 65,98 & 296,58 \\
\hline 4 & 2701,62 & 474,25 & 1641,63 & 367,70 & 1037,23 & 296,58 \\
\hline
\end{tabular}

Cuadro 10. Valor del suelo en base a dos alternativas de uso y a diferente. Tasa guía de interés. (TGI), para cuatro casos. 
Figura 1. Valor del suelo con uso forestal Caso 4 y uso tradicional ganadero.

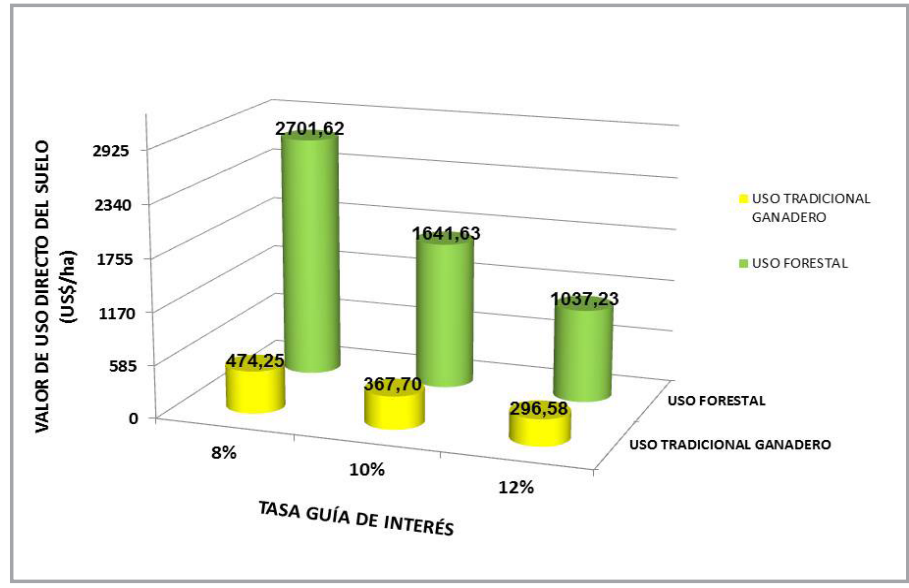

En la Figura 1, se muestra la comparación entre el valor del suelo con el uso tradicional ganadero y el valor del suelo con uso forestal, considerando el Caso 4, es decir, incluyendo como producto a la madera en pie, hongos comestibles, servicio turístico y servicio de secuestro de carbono. En el cual se observa claramente la ventaja del uso forestal sobre el uso tradicional.

\section{Discusión}

El costo de establecimiento en el caso del presente estudio, se encuentra por debajo de los montos que INIA (2014) y CONIF (2013) señalan; esto estaría relacionado con las diferencias que pueden haber entre el PPF y otros proyectos, en cuanto a la tecnología empleada y en cuanto a los costos de transporte de los plantones en la etapa inicial, teniendo en cuenta que el PPF, contaba con dos viveros forestales in situ de gran producción.

El monto de los gastos anuales de mantenimiento que se ha estimado en este estudio, es considerablemente menor de los montos que Fernández et al. (2012) y Novoa (2005) señalan. Esto tendría que ver con el hecho de que en otros países, el manejo de plantaciones forestales es más intensivo y planificado, por lo mismo, sus gastos de mantenimiento abarcan costos que no se han considerado en esta investigación, ya sea porque no se encontraron registros o porque son gastos que no se han realizado durante el mantenimiento anual de las plantaciones del PPF.

En el caso del ingreso capitalizado se observa que son similares con las tres tasas de interés consideradas, para los tres primeros casos; en el cuarto caso, al incluir el servicio de secuestro de carbono el ingreso capitalizado se incrementa significativamente, al aumentar la tasa de interés; lo cual pone en evidencia la importancia del servicio de secuestro de carbono en la valoración del recurso forestal.

Se observa que en la medida que se incrementan los bienes y servicios que se producen con el uso del suelo forestal, su valor potencial se incrementa, este incremento es muy notorio cuando se incluye el servicio de secuestro de carbono.

El valor del suelo obtenido, según Prieto et al. (1998) y Ortuño et al. (2007), es lo esperado, ya que el valor del suelo es muy sensible a la tasa de interés, por el largo periodo de maduración del producto principal (madera en pie).

En referencia al Cuadro 5, para el Caso 1, el valor del suelo es positivo para las tres TGI; sin embargo, son valores menores que el valor máximo encontrado en Colombia por Restrepo et al. (2012); estas diferencias en el valor del suelo, podrían ser explicadas por las diferencias de duración del turno y la tasa de interés que se ha empleado en cada estudio. 
Según el plan original del PPF, las plantaciones de Granja Porcón fueron establecidas con el objetivo de proveer materia prima para la industria de pulpa y papel; sin embargo, por razones de carácter técnico, hubo un cambio de objetivo y actualmente la producción se orienta a la industria de transformación mecánica; esto también habría influido en los resultados obtenidos, debido a que las labores silviculturales necesarias para el nuevo objetivo de la plantación, no se realizaron en el momento oportuno, lo cual influyó desfavorablemente en el volumen y la calidad de la madera en pie; lo cual tiene incidencia directa en el valor potencial del suelo.

La escasa influencia de la variación de la TGI sobre la renta del suelo, se debe a que el ciclo de producción es anual, al igual que el periodo de capitalización de la TGI; y a que la TGI sólo afecta al costo del capital. Asimismo, la relación inversa entre el valor de uso directo del suelo y la tasa guía de interés guarda relación con el hecho de que el valor del suelo ha sido estimado como la actualización de una serie anual perpetua; donde la TGI es el denominador de la relación.

Basados en este análisis comparativo, es evidente que la actividad forestal en la zona de Cajamarca, a través del establecimiento de plantaciones forestales de pinos, ha generado un impacto económico positivo sobre el valor de uso directo del suelo, al incrementar dicho valor con respecto a la actividad ganadera tradicional de crianza extensiva de ovinos; por lo tanto, el uso forestal se afirma como la mejor alternativa de uso del suelo en el ámbito de estudio.

\section{Conclusiones}

En las condiciones actuales de uso del suelo forestal, en la zona de estudio, es decir, con plantaciones de pinos y considerando la producción de madera rolliza en pie, hongos comestibles y servicios de ecoturismo; el valor del suelo con el uso forestal es mayor que el valor del suelo con el uso tradicional de ganadería extensiva, para tasas guías de interés de $8 \%$ y
10\%; con una relación inversa, es decir, el valor del suelo disminuye al aumentar la tasa guía de interés.

Para el uso forestal, al incluir el servicio de secuestro de carbono, como producción de la plantación forestal de pinos, el valor de uso directo del suelo se incrementa significativamente; y se hace mucho mayor que el correspondiente valor del suelo con el uso tradicional. Esta ventaja en el valor del suelo disminuye, en la medida que la tasa guía de interés aumenta.

Para tasas guías de interés menores o igual a $10 \%$, la mejor alternativa de uso del suelo en la zona de estudio, es establecer plantaciones forestales de pinos; inclusive considerando como producto de la plantación solo a la madera en pie.

Para una tasa guía de interés mayor o igual a $12 \%$, la alternativa del uso forestal será mejor que la del uso tradicional de ganadería extensiva, sólo si se incluye en la producción de la plantación forestal a: madera rolliza en pie, hongos comestibles y los servicios de ecoturismo y secuestro de carbono.

\section{Agradecimientos}

Los autores expresan su agradecimiento a la Asociación Civil para la Investigación y Desarrollo Forestal (ADEFOR) Cajamarca Perú. Por las facilidades brindadas, para la realización del presente estudio.

\section{Bibliografía}

Asociación Civil para la Investigación y Desarrollo Forestal (ADEFOR). 1993. Estudio de Caso Granja Porcón. Cajamarca, PE. s.p.

Asociación Civil para la Investigación y Desarrollo Forestal (ADEFOR). 1998. Valorización de plantaciones forestales industriales en Cajamarca de propiedad compartida con SPL: Informe final. Cajamarca, PE. s.p.

Asociación Civil para la Investigación y Desarrollo Forestal (ADEFOR). 2005. Plan General de Manejo Forestal de las Plantaciones de la Cooperativa Agraria Atahualpa Jerusalén de 
Trabajadores LTDA - Granja Porcón, Establecido en convenio con el Proyecto Piloto de Forestación (PPF). Cajamarca, PE. 87 p.

Alegre, J; Arévalo, L; Ricse, A; Callo-Concha, D; Palm, C. 2004. Sistemas Agroflorestais, tendencia da Agricultura Ecológica nos trópicos: secuestramiento de carbono con sistemas alternativos en el Perú. Eds: M Muller; A Da Gama; I Fontes; M Fernández. s.n.t. 292 p. (Serie: $27-32)$.

Carton, Ch. 2000. Impacto de la reforestación en comunidades campesinas de los Andes peruanos. Lima, PE, s.e. 194 p.

Chidiak, M; Moreyra, A; Greco, C. 2003. Captura de carbono y desarrollo forestal sustentable en la Patagonia Argentina: Sinergias y desafíos. Buenos Aires, AR, s.e. 118 p.

Cooperativa Agraria Atahualpa Jerusalén de Trabajadores (CAT). 2009. Cooperativa Agraria "Atahualpa Jerusalén": Granja Porcón (diapositivas). Cajamarca, PE. 35 diapositivas, color.

Corporación Nacional de Investigación y Fomento Forestal (CONIF). 2013. Estudio de Costos de las Especies Forestales beneficiarias del CIF: Informe final. Bogotá, CO. 156 p.

Dirección Regional de Comercio Exterior y Turismo (DIRCETUR). 2006. Plan Estratégico Regional de Turismo de Cajamarca 2006 2015. Cajamarca, PE. 40 p.

Fernández, M; Barroetaveña, C; Bassani, V; Ríos, F. 2012. Rentabilidad del aprovechamiento del hongo comestible Suillus luteus para productores forestales y para familias rurales de la zona cordillerana de la provincia del Chubut, Argentina. Bosque 33(1):43-52.

Fundación Romero, PE. 2009. Buenas Nuevas: Hongos y Pinos - parte 3 (video). Marayhuaca Lambayeque, PE. 1 video (9 min., 30 seg.), son., color.

García, M. 1999. Evaluación de la producción natural de hongos comestibles en el predio Granja Porcón con énfasis en la especie Suillus luteus en plantaciones de Pinus patula. Tesis Ing. Forestal. Lima, PE, UNALM. 107 p.
Instituto Nacional de Innovación Agraria (INIA). 2014. INIA lanza tecnología de sinergia para producción rentable de pino y café (en línea). Lima, PE. Consultado 15 abr. 2015. Disponible en http:// www.inia.gob.pe/SALA-DE-PRENSA/NOTAS-DE-PRENSA/695-INIA-LANZA-TECNOLOGIA-DE-SINERGIA-PARA-PRODUCCION-RENTABLE-DE-PINO-Y-CAFE

Klemperer, D. 2003. Forest resource economics and finance. Paperback edition published by W. David Klemperer. Printed in the United States of America. $551 \mathrm{p}$.

Ministerio de Agricultura (MINAG). 1992. Primer compendio estadístico agrario 1950 1991. Ed. Oficina de Estadística Agraria. Lima, PE. 1365 p.

Ministerio de Agricultura (MINAG). 2013. Perú Forestal en números: año 2012. Ed. DGFFS. Lima, PE. 209 p.

Ministerio de Comercio Exterior y Turismo (MINCETUR). 2013. Investigación de mercados turísticos: Cajamarca (en línea). Consultado 25 abr. 2014. Disponible en http://www. peru.travel/impp/

Novoa, R. 2005. Evaluación económica de cuatro regímenes silviculturales intensivos en plantaciones experimentales de Pinus radiata D. Don. Jauja, Comuna de Calipulli (IX Región). Tesis Ing. Forestal. Valdivia, CL, Universidad Austral de Chile. 65 p.

Organización de las Naciones Unidas para la Alimentación y la Agricultura (FAO). 2004. Informe Nacional Perú (en línea). Consultado 28 dic. 2013. Disponible en http://www.fao.org/ docrep/007/j4024s/j4024s00.htm

Ortuño, S; Madrigal, A; González, I. 2007. Apuntes de Valoración Agraria y Forestal. Madrid, ES, Universidad Politécnica de Madrid. $103 \mathrm{p}$.

Petriceks, J. 1981. Apuntes de Economía y Valoración Forestal. Mérida, VE, Universidad de los Andes. $126 \mathrm{p}$.

Prieto, A; Díaz, L; Hernando, A. 1998. Valoración de montes arbolados. Catastro N. ${ }^{\circ}$ 33:65-82. 
Pro Chile. 2012. Estudio de mercado de Bonos de Carbono. Berlín, DE. 31 p.

Restrepo, H; Orrego, S; Del Valle, J; Salazar, J. 2012. Rendimiento, turno óptimo forestal y rentabilidad de plantaciones forestales de Tectona grandis y Pinus patula en Colombia. Interciencia 37(1):14-20.

Villanueva, M. 1994. Incidencia de los precios, el crédito y el tipo de cambio en la producción de lana de Ovino y fibra de Alpaca. Tesis Economista. Lima, PE, UNALM. 116 p.

Presentado: $29 / 06 / 2016$

Aceptado: 03/08/2017 